\title{
Avaliação da variação de pH e da permeabilidade da dentina cervical em dentes submetidos ao tratamento clareador $^{\dagger}$
}

\section{Evaluation of $\mathrm{pH}$ variation and cervical dentin permeability in teeth submitted to bleaching treatment}

\author{
Mariela S. G. Dezotti* \\ Mário Honorato Silva e Souza Júnior** \\ Celso Kenji Nishiyama***
}

\begin{abstract}
RESUMO: A reabsorção cervical externa da raiz é uma das desvantagens do procedimento clareador. Vários são os mecanismos que podem ser responsáveis por desencadear esta reabsorção, dentre eles, a ação química e física dos materiais utilizados, bem como a morfologia da junção amelocementária. Este trabalho teve como objetivo observar uma possivel via de comunicação entre a câmara pulpar e a superficie externa da raiz, medindo o pH e a infiltração de corante na dentina cervical após o procedimento clareador. Realizou-se o tratamento endodôntico em 34 dentes incisivos permanentes. Os dentes foram divididos em 3 grupos experimentais de acordo com o nível do corte da obturação e selamento da embocadura dos canais com cimento de ionômero de vidro. O clareamento foi realizado usando perborato de sódio e peróxido de hidrogênio a 30\%. As leituras do $\mathrm{pH}$ foram realizadas após $30 \mathrm{~min}, 24$ h, 48 h e 72 h do início do procedimento. A seguir, os dentes foram imersos em fucsina básica a 0,5\% por $24 \mathrm{~h}$ para determinarmos possiveis diferenças na permeabilidade da dentina cervical. Os resultados mostraram que o $\mathrm{pH}$ apresentou tendência a se modificar quando o corte da obturação permaneceu na embocadura dos canais, bem como quando se removeram $2 \mathrm{~mm}$ da obturação e quando se selou a embocadura com cimento de ionômero de vidro. A permeabilidade dentinária aumentou nos 3 grupos experimentais, em comparação com os dentes que compreenderam o grupo controle. Estas leves diferenças podem sugerir uma via de comunicação entre a câmara pulpar e a superfície externa da raiz.

UNITERMOS: Clareamento de dente; Reabsorção de dente; Peróxido de hidrogênio.
\end{abstract}

\begin{abstract}
External cervical root resorption is one of the disadvantages of the bleaching procedure. There are several mechanisms that may be responsible for causing resorption, such as the chemical and physical action of the utilized materials and the morphology of the cementoenamel junction. The purpose of this study was to investigate the presence of a communication between the pulp chamber and the external root surface. The investigation was carried out by means of $\mathrm{pH}$ tests and measurement of dye infiltration into cervical dentin after the bleaching procedure. Thirty-four human permanent incisors were submitted to endodontic treatment. The teeth were assigned to three experimental groups, according to the level at which the filling was cut, and to the sealing of the root canal entrance with glass ionomer cement. Sodium perborate and 30\% hydrogen peroxide were utilized for bleaching. pH readings were carried out after 30 minutes, $24 \mathrm{~h}, 48 \mathrm{~h}$ and $72 \mathrm{~h}$ from the beginning of the procedure. The teeth were immersed in $0.5 \%$ basic fuchsin for $24 \mathrm{~h}$ in order to determine possible differences in the permeability of cervical dentin. The results revealed that $\mathrm{pH}$ tended to change when the root filling was cut at the entrance of the canal, when $2 \mathrm{~mm}$ of the filling were removed, and when the canal entrance was sealed with glass ionomer. Dentinal permeability increased in the three experimental groups, in comparison with the control group. These slight differences may suggest a communication between the pulp chamber and the external root surface.
\end{abstract}

UNITERMS: Tooth bleaching; Tooth resorption; Hydrogen peroxide.

\section{INTRODUÇÃO}

A estética constitui importante papel na Odontologia moderna. A beleza dos dentes é influenciada pelo contorno, forma, simetria e coloração ${ }^{14}$.
Sabe-se que dentes submetidos ao tratamento endodôntico podem sofrer mudanças em sua cor. O material necrótico no interior dos canais, observado em necroses e gangrenas pulpares, sofre degradação protéica, a hemólise, e seus produtos,

$\dagger$ Parte da Monografia de Especialização em Endodontia.

*Mestre e Doutoranda em Estomatologia; **Professor Adjunto - Departamento de Dentística Restauradora da Faculdade de

Odontologia de Bauru da Universidade de São Paulo.

*** Chefe do Setor de Endodontia do Hospital de Reabilitação de Anomalias Craniofaciais da Universidade de São Paulo

(HRAC-USP). Coordenador dos Cursos de Aperfeiçoamento e Especialização em Endodontia da Profis, Bauru - SP. 
Dezotti MSG, Silva e Souza Júnior MH, Nishiyama CK. Avaliação da variação de pH e da permeabilidade da dentina cervical em dentes submetidos ao tratamento clareador. Pesqui Odontol Bras 2002;16(3):263-268.

penetrando nos túbulos dentinários, podem resultar no escurecimento dentário. Outras causas, como o uso de materiais contendo prata precipitada ou iodofórmio, abertura coronária incorreta, sangramento abundante em pulpectomia e permanência de materiais obturadores na câmara pulpar, podem causar o escurecimento da estrutura dentária ${ }^{15}$.

O tratamento que devolve ao dente sua cor e translucidez originais é denominado clareamento dentário, preferido por pacientes e profissionais, por conservar os dentes naturais, ao contrário dos tratamentos protéticos como facetas ou próteses, que os desgastam.

O clareamento dentário é baseado na liberação de oxigênio nascente e, para prevenir ou atenuar complicações desnecessárias, requer um número de precauções, entre elas: a presença de obturação endodôntica bem condensada, para prevenir uma irritação periapical retrógrada, devido à infiltração dos materiais clareadores e a proteção do tecido periodontal devido à causticidade dos mesmos.

Duas técnicas têm sido propostas, a "walking bleach", bem aceita por proporcionar menor tempo de permanência na cadeira do consultório odontológico, e a termocatalítica, sendo que a diferença básica entre elas é a maneira pela qual o oxigênio nascente é liberado para a limpeza química e mecânica dos canalículos dentinários ${ }^{15}$. Uma pasta de perborato de sódio e água destilada é selada na câmara pulpar, permanecendo por 4 a 7 dias, quando o paciente retorna ao consultório e observa-se a alteração da cor e a necessidade de nova sessão.

Foi proposta a substituição da água pelo peróxido de hidrogênio, pois desta maneira a liberação de oxigênio seria mais eficiente ${ }^{15}$. Preconizou-se ainda o emprego do ultra-som como agente coadjuvante para o clareamento dentário ${ }^{14}$.

A partir da década de 70 , iniciaram-se avaliações mais criteriosas quanto ao emprego de peróxido de hidrogênio, indicado desde 1895. Alguns trabalhos mostraram reabsorções cervicais externas, em decorrência de seu uso $\mathrm{s}^{3,4,6,7,9,10,11}$. A reabsorção cervical externa pode ocorrer após um trauma agudo ou crônico, reimplante dentário, ou ser idiopática. Apesar de alguns estudos constatarem reabsorções após o clareamento, ainda não se pode estabelecer uma relação causa-efeito definitiva neste processo ${ }^{13}$. Vários mecanismos relacionados ao clareamento dentário podem estar ligados ao processo de reabsorção: a morfologia da junção amelocementária $^{13}$, a redução da dureza dos teci- dos mineralizados pela degradação dos componentes orgânicos e inorgânicos ${ }^{18}$, a difusibilidade dos agentes clareadores pelos túbulos dentinários, modificação do $\mathrm{pH}$ na superficie radicular cervical externa $^{5,8,17,21}$ e inflamação na área cervical ${ }^{3,6,10,20}$.

Em virtude desta propriedade dos materiais clareadores (relação causa-efeito com a reabsorção cervical externa), nos propusemos a observar uma possivel comunicação entre a câmara pulpar e a superficie externa da raiz, medindo o $\mathrm{pH}$ da água em que os dentes permaneceram imersos após o clareamento e a infiltração de corante pelos túbulos dentinários cervicais.

\section{MATERIAIS E MÉTODOS}

Foram utilizados 34 incisivos extraídos em um tempo médio de 60 dias. Os dentes foram limpos com curetas periodontais, a fim de remover todo o ligamento periodontal e qualquer detrito que neles estivessem contidos. Quando observamos defeitos na junção amelocementária (JAC), os dentes foram descartados. Realizou-se tratamento endodôntico e obturação com cimento Sealer 26 e os dentes foram divididos em 4 grupos: Grupo 1 - 9 dentes tiveram o corte da obturação realizado com instrumento aquecido, $2 \mathrm{~mm}$ abaixo da JAC vestibular; Grupo 2 - 9 dentes tiveram o corte da obturação até o nível da JAC vestibular; Grupo 3 - 8 dentes tiveram o corte da obturação $2 \mathrm{~mm}$ abaixo da JAC vestibular e esta foi selada com cimento de ionômero de vidro Vitrebond da 3M; Grupo 4 - 8 dentes serviram como grupo controle (não receberam o curativo com a pasta clareadora). Preparou-se uma pasta de perborato de sódio (2 g) e peróxido de hidrogênio a $30 \%(1 \mathrm{ml})$, que foi selada dentro da câmara pulpar de cada dente com resina composta Herculite. Foi realizada uma proteção dos dentes com esmalte de unhas, com exceção dos $4 \mathrm{~mm}$ na área da JAC, por onde observariamos provável passagem dos agentes clareadores para a água destilada, alterando o $\mathrm{pH}$ da mesma. Os dentes foram imersos em água destilada com $\mathrm{pH} 5,6$. As leituras do $\mathrm{pH}$ foram realizadas em um pHmetro modelo B371 aos $30 \mathrm{~min}, 24 \mathrm{~h}, 48 \mathrm{~h}$ e $72 \mathrm{~h}$ após a colocação do curativo. Os dados obtidos foram submetidos à análise de variância (ANOVA), seguida de um teste de comparações múltiplas (Tukey).

Em seguida, o curativo foi removido do acesso coronário e os dentes foram novamente protegidos com esmalte de unhas, à exceção dos $4 \mathrm{~mm}$ da área da JAC. Colocou-se na câmara pulpar uma bolinha de algodão embebida em fucsina básica a 
Dezotti MSG, Silva e Souza Júnior MH, Nishiyama CK. Avaliação da variação de pH e da permeabilidade da dentina cervical em dentes submetidos ao tratamento clareador. Pesqui Odontol Bras 2002;16(3):263-268.

$0,5 \%$, e então os dentes foram imersos neste mesmo corante e aí deixados por $24 \mathrm{~h}$. Realizou-se lavagem em água corrente por $24 \mathrm{~h}$. Os dentes foram secos e incluídos em resina acrílica e cortados no sentido vestíbulo-lingual no aparelho Labcut 1010, com o objetivo de medir a infiltração cervical de corante, mostrando o aumento da permeabilidade da dentina. Os dentes foram avaliados segundo os escores: 0) nenhuma infiltração; 1) infiltração até a metade da espessura de dentina, e 2) infiltração envolvendo toda a espessura de dentina. Os dados foram submetidos a um teste não-paramétrico (Kruskal-Wallis), seguido de um teste de comparações múltiplas dos grupos.

Foram realizadas leituras do $\mathrm{pH}$ de todas as soluções usadas no experimento: o pH da água destilada; do perborato de sódio misturado com água, apenas como comparação; do perborato de sódio misturado ao peróxido de hidrogênio a $30 \%$ e do peróxido de hidrogênio a $30 \%$.

\section{RESULTADOS}

Os Gráficos 1, 2 e 3 mostram as leituras de $\mathrm{pH}$ realizadas nos 3 grupos experimentais, respectivamente, sempre comparando-se com o $\mathrm{pH}$ da água destilada pura.

No Gráfico 1, observamos que, após $30 \mathrm{~min}$, o $\mathrm{pH}$ da água se elevou para 6,4 , em $24 \mathrm{~h}$ foi para 6,6 , em $48 \mathrm{~h}$, baixou para $6,3 \mathrm{e}$ em $72 \mathrm{~h}$ ficou em 6,6 . No Gráfico 2, observou-se que, após $30 \mathrm{~min}$, o $\mathrm{pH}$ se elevou para 7,3 , em $24 \mathrm{~h}$ ficou em 7,2 , em $48 \mathrm{~h}$, em 7,1 e em $72 \mathrm{~h}$, em 7,2 . As leituras de $\mathrm{pH}$ realizadas no Grupo 3 (Gráfico 3) mostraram que, aos $30 \mathrm{~min}$, o pH passou para 6,6, em $24 \mathrm{~h} \mathrm{o} \mathrm{pH} \mathrm{fi-}$ cou em 7,1 , após 48 h, estava em 6,8 e em 72 h ficou em 7,1. O Gráfico 4 mostra as leituras de $\mathrm{pH}$ do grupo 4 (controle). Observa-se que o $\mathrm{pH}$ praticamente não se alterou, mostrando-se, aos 30 min em 5,6, em 24 h, 5,3, em 48 h, 5,3 e em 72 h, 5,2. A análise estatística mostrou que houve diferença significante entre o $\mathrm{pH}$ dos 4 grupos $(p \geq 0,0000001)$. Não houve diferença estatística no $\mathrm{pH}$ em relação ao tempo avaliado $(\mathrm{p}=0,783756)$. Pelo teste Tukey concluiu-se que houve diferença em nivel de $5 \%$ de significância entre os Grupos: 1 e 4; 2 e 4; e 3 e 4.

No Gráfico 5, observamos a infiltração de corante nos grupos experimentais e no grupo controle, sendo que este mostrou uma infiltração de 0,3 , o Grupo 1, de 0,8, o Grupo 2, de 0,7 e o Grupo 3, de 0,8 . Notou-se aumento da permeabilidade dentinária em todos os dentes do grupo experimental,

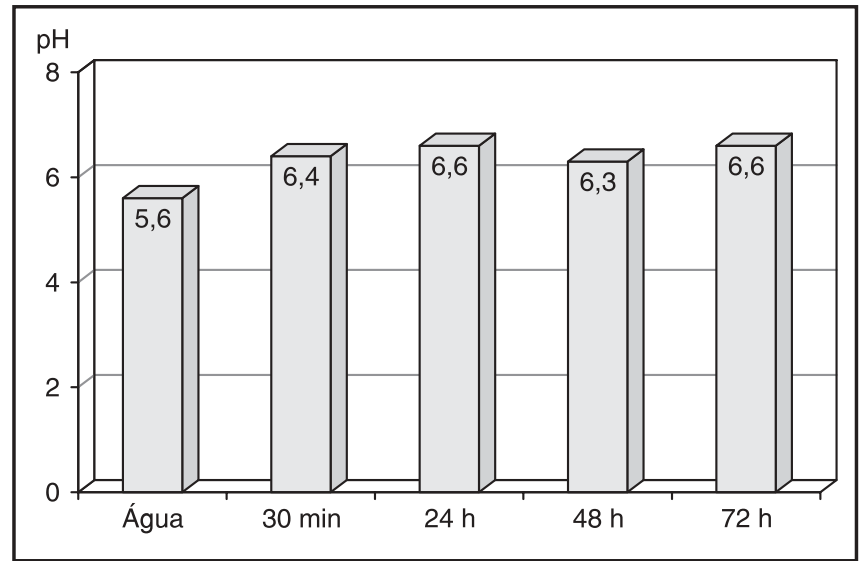

GRÁFICO 1 - Leitura do $\mathrm{pH}$ realizada no Grupo $1 \mathrm{em}$ comparação com o pH da água destilada pura, após 30 minutos, 24 h, 48 h e 72 h da colocação do curativo de perborato de sódio e peróxido de hidrogênio a 30\%.

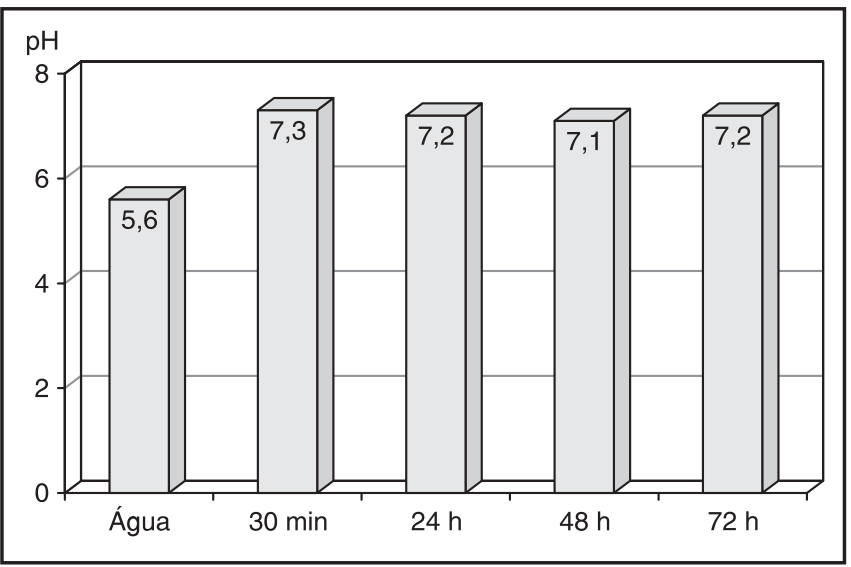

GRÁFICO 2 - Leitura do $\mathrm{pH}$ realizada no Grupo 2 em comparação com o pH da água destilada pura, após 30 minutos, 24 h, 48 h e 72 h da colocação do curativo de perborato de sódio e peróxido de hidrogênio a 30\%.

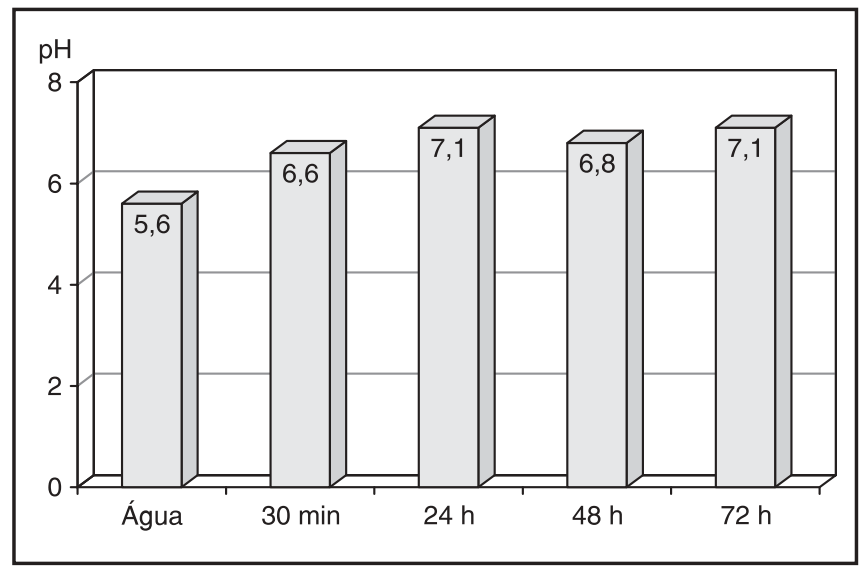

GRÁFICO 3 - Leitura do $\mathrm{pH}$ realizada no Grupo $3 \mathrm{em}$ comparação com o pH da água destilada pura, após 30 minutos, $24 \mathrm{~h}, 48 \mathrm{~h}$ e $72 \mathrm{~h}$ da colocação do curativo de perborato de sódio e peróxido de hidrogênio a 30\%. 
Dezotti MSG, Silva e Souza Júnior MH, Nishiyama CK. Avaliação da variação de pH e da permeabilidade da dentina cervical em dentes submetidos ao tratamento clareador. Pesqui Odontol Bras 2002;16(3):263-268.

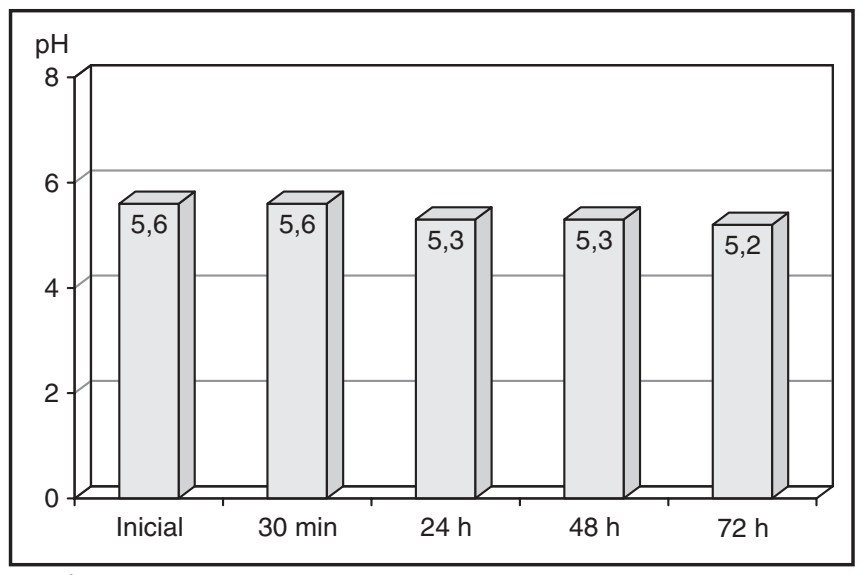

GRÁFICO 4 - Leitura do pH realizada no Grupo 4 (controle) em comparação com o pH inicial da água destilada pura, após 30 minutos, 24 h, 48 h e 72 h.

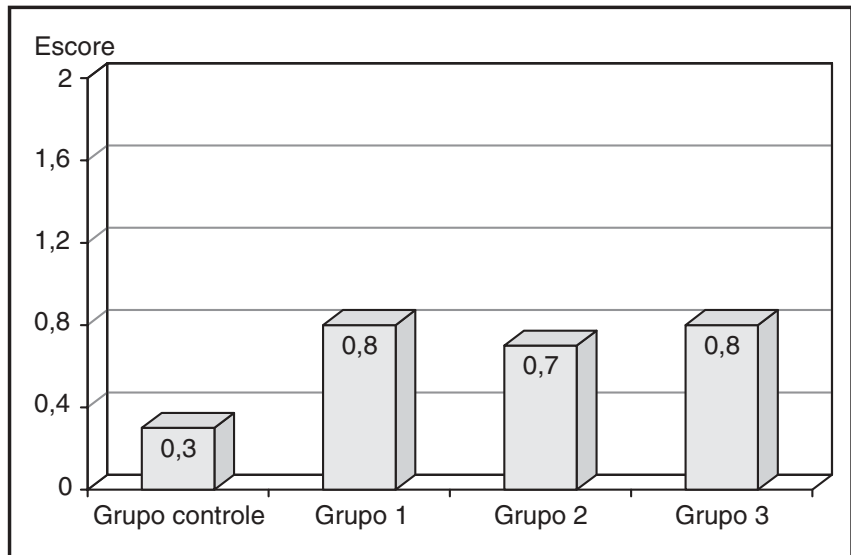

GRÁFICO 5 - Infiltração de corante no grupo controle e nos grupos experimentais.

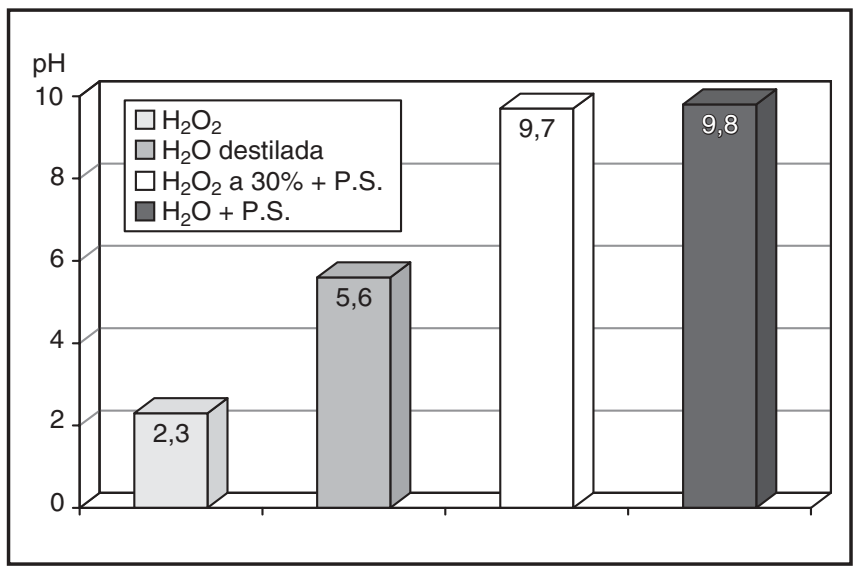

GRÁFICO 6 - Valores obtidos com as leituras de pH do peróxido de hidrogênio puro a $30 \%\left(\mathrm{H}_{2} \mathrm{O}_{2}\right.$ a $\left.30 \%\right)$, da água destilada pura $\left(\mathrm{H}_{2} \mathrm{O}\right)$, do peróxido de hidrogênio a $30 \%\left(\mathrm{H}_{2} \mathrm{O}_{2}\right.$ a $\left.30 \%\right)$ misturado com perborato de sódio (P.S.) e da água destilada $\left(\mathrm{H}_{2} \mathrm{O}\right)$, misturada ao perborato de sódio (P.S.). em relação ao grupo controle, sendo que esta foi observada da câmara pulpar para a superficie externa da raiz. O teste de análise de variância não-paramétrico (Kruskal-Wallis) mostrou que houve diferença significante entre os grupos $(\mathrm{H}=11,411,3$ graus de liberdade, e $\mathrm{p} \geq 0,010)$. $\mathrm{O}$ teste de comparações múltiplas dos Grupos 1, 2, 3, versus Grupo 4 mostrou que houve diferença entre o Grupo 3 e 4 e entre o Grupo 1 e 4 (com $\mathrm{p} \geq 0,05)$.

Os valores obtidos com as leituras de $\mathrm{pH}$ da água destilada, do perborato de sódio misturado com a água destilada, do perborato de sódio misturado com o peróxido de hidrogênio a $30 \%$ e peróxido de hidrogênio a $30 \%$ puro, podem ser observados no Gráfico 6. Verifica-se a acidez do peróxido de hidrogênio a $30 \%$ puro $(2,3)$ e a alcalinidade da mistura obtida com perborato de sódio e água destilada $(9,8)$, permanecendo a mistura de perborato de sódio com peróxido de hidrogênio com valores bem próximos $(9,7)$. Observa-se também o $\mathrm{pH}$ da água destilada, que serviu como controle em todos os passos do experimento.

\section{DISCUSSÃO}

É aceitável a hipótese de que o clareamento dentário resulte em reabsorção externa da raiz, porém é necessário analisar todos os fatores etiológicos responsáveis por este fenômeno.

O peróxido de hidrogênio a $30 \%$ e o perborato de sódio são os agentes clareadores mais usados na prática odontológica, e têm sido freqüentemente relacionados com o desenvolvimento de reabsorções cervicais externas ${ }^{6,7}$.

Alguns estudos mostraram que as pastas clareadoras são alcalinas, e que sua alcalinidade aumenta de acordo com o tempo e diluente utiliza$\mathrm{do}^{5,17}$. O aumento do $\mathrm{pH}$ observado com o tempo, pode ser devido ao fato de que o peróxido de hidrogênio, ácido, libera nesta reação, água e oxigênio. Por 14 dias, a maioria do peróxido de hidrogênio é decomposto e o $\mathrm{pH}$ da mistura pode se igualar àquele do perborato de sódio misturado com a água ${ }^{17}$.

Assume-se que a destruição na área cervical da raiz não é resultante do $\mathrm{pH}$ ácido, mas sim, de injúria direta às estruturas vitais ao redor da raiz $^{17}$. O presente trabalho mostrou que o $\mathrm{pH}$ da pasta obtida com $2 \mathrm{~g}$ de pó de perborato de sódio e $1 \mathrm{ml}$ de líquido de peróxido de hidrogênio a 30\%, é alcalino. Nossos resultados concordam com Rotstein, Friedman ${ }^{17}$ em 1991, porém, não observa- 
Dezotti MSG, Silva e Souza Júnior MH, Nishiyama CK. Avaliação da variação de pH e da permeabilidade da dentina cervical em dentes submetidos ao tratamento clareador. Pesqui Odontol Bras 2002;16(3):263-268.

mos aumento da alcalinidade com o passar do tempo. A análise de variância (ANOVA) mostrou diferença estatisticamente significante entre os grupos ( $\mathrm{p} \geq 0,0000001)$, mas não, entre os tempos $(\mathrm{p}=0,783756)$.

Defeitos cementários, principalmente na JAC, aumentam significativamente a infiltração dos agentes clareadores para a dentina cervical $^{19}$. Estes defeitos podem estar presentes em aproximadamente $25 \%$ da população ${ }^{12}$. A auto-imunidade da dentina tem um aspecto significante, pois esta apresenta proteínas específicas, muitas vezes, inacessiveis às células de reconhecimento imunológico humano. Sua organização dificulta a exposição direta das proteínas não colagênicas à ação das células processadoras de antígenos, permanecendo as proteínas dentinárias incorporadas em uma matriz mineralizada e atuando como antígenos seqüestrados. Quando expostas, não são reconhecidas como próprias pelo organismo, e desencadeiam resposta específica, representada por mobilização celular, com o intuito de efetivar a eliminação dos antígenos, enquanto os clastos atuam como o principal efetor. O mecanismo gerador do processo reabsortivo requer a presença de fatores locais, como a liberação de citocinas, para atuar como promotor de células clásticas. A forma irregular da JAC colabora na identificação desta área como predisposta à instalação das reabsorções cervicais externas, frente à ação de determinados fatores tais como: agentes clareadores, traumatismos e movimentação dentária induzida $^{13}$.

Estamos de acordo com a literatura, visto que observamos a difusão dos materiais clareadores por meio da dentina radicular cervical, quando obtivemos um aumento do $\mathrm{pH}$ da água em que os dentes foram colocados, à exceção do grupo controle.

Bases protetoras colocadas na superficie interna da dentina radicular podem reduzir a ocorrência de reabsorção cervical externa da raiz, impedindo a infiltração de materiais clareadores da câmara pulpar para a superficie externa radicular ${ }^{4,9,19}$. Sugere-se que uma barreira de $2 \mathrm{~mm}$ deve ser colocada apicalmente a $\mathrm{JAC}^{2,9}$. Os resultados obtidos por Rotstein, Friendman ${ }^{17}$ (1991) demonstraram que todos os materiais utilizados como base protetora (óxido de zinco e eugenol, IRM, resina composta e cimento de ionômero de vidro) foram igualmente eficazes na prevenção da infiltração radicular de peróxido de hidrogênio, quando a espessura desta base excedia $1 \mathrm{~mm}$, o que não encontramos em nosso experimento. Nenhum dos materiais utilizados (guta-percha e ionômero de vidro) foi eficaz para impedir a infiltração dos materiais clareadores da câmara pulpar para a superficie externa da raiz, sendo que em todos os grupos houve diferença estatisticamente significante em relação ao grupo controle, isto é, houve a passagem dos agentes clareadores para a água destilada, aumentando o seu pH. Não houve diferença estatística entre os grupos, indicando uma ou outra barreira, como mais ou menos eficiente.

Em relação à permeabilidade dentinária, somos concordes com alguns relatos da literatura, ${ }^{1,16}$, em que se observou aumento da mesma em relação ao grupo controle. Os resultados demonstraram, pelo teste de análise de variância não-paramétrico (Kruskal-Wallis), diferença estatisticamente significante entre os grupos $(\mathrm{H}=11,411,3$ graus de liberdade, e $p \geq 0,010$ ). O teste de comparações múltiplas dos Grupos 1, 2 e 3 versus o Grupo 4 mostrou que houve diferença entre o Grupo 3 e Grupo 4, e Grupo 1 e Grupo 4 (p $\geq 0,05$ ). Não foi demonstrada diferença significante entre os Grupos 2 e 4 .

\section{CONCLUSÕES}

Tendo em vista os resultados obtidos concluímos que: existe comunicação entre a câmara pulpar e a superficie externa da raiz, uma vez que observamos aumento do $\mathrm{pH}$ da água em que os espécimes foram incluídos; os agentes clareadores aumentam a permeabilidade dentinária, constatado por meio de maior infiltração de corante nos grupos experimentais; as bases protetoras estudadas não foram eficazes na prevenção da passagem dos agentes clareadores da câmara pulpar para a superficie externa da raiz.

\section{AGRADECIMENTOS}

Agradecemos à CAPES pelo suporte financeiro para realização deste trabalho. 
Dezotti MSG, Silva e Souza Júnior MH, Nishiyama CK. Avaliação da variação de pH e da permeabilidade da dentina cervical em dentes submetidos ao tratamento clareador. Pesqui Odontol Bras 2002;16(3):263-268.

\section{REFERÊNCIAS}

1. Barbosa SV, Safavi KE, Spanberg LSW. Influence of sodium hypoclorite on the permeability and structure of cervical human dentine. Int Endod $\mathrm{J}$ 1994;27(6):309-12.

2. Costas FL, Wong M. Intracoronal isolating barriers: effect of location on root leakage and effectiveness of bleaching agents. J Endod 1991;17(8):365-8.

3. Cvek M, Lindvall AM. External root resorption following bleaching of pulpless teeth with oxigen peroxide. Endod Dent Traumatol 1985;1(2):56-60.

4. Friendman S, Rotstein I, Libfeld H, Stabholz A, Heling I. Incidence of external root resorption and esthetic results in 58 bleached pulpless teeth. Endod Dent Traumatol 1988;4(1):23-6.

5. Fuss Z, Szajkis S, Tagger M. Tubular permeability to calcium hydroxide and to bleaching agents. J Endod 1989;15(8):362-4.

6. Harrington GW, Natkin E. External resorption associated with bleaching of pulpless teeth. J Endod 1979;5(11):344-8.

7. Heithersay GS, Dahistrom SW, Marin PD. Incidence of invasive cervical resorption in bleached root-filled teeth. Aust Dent J 1994;39(2):82-7.

8. Kehoe JC. pH reversal folowing in vitro bleaching of pulpless teeth. J Endod 1987;13(1):6-9.

9. Lado EA. Bleaching of endodontically treated teeth: an update on cervical resorption. Gen Dent 1988;36(6):500-1.

10. Madison S, Walton R. Cervical root resorption following bleaching of endodontically treated teeth. J Endod 1990;16(12):570-4.

11. Montgomery S. External cervical resorption after bleaching a pulpless tooth. Oral Surg 1984;57(2):203-6.
12. Müller C, Van Wyk CW. The amelocemental junction. J Dent Assoc S Afr 1984;39(12):799-803.

13. Neuvald LR. Análise microscópica da junção amelocementária com ênfase para os mecanismos envolvidos nas reabsorções cervicais externas. Bauru [Dissertação de Mestrado]. Bauru: Faculdade de Odontologia da USP; 1997.

14. Nishiyama CK, Lacerda AG, Souza Júnior MH, Francischone CE, Ishikiriama A, Berbert A. Blanchiment des dents depulpees avec assistance ultrasonique. Rev Fr Endod 1989;8(1):43-7.

15. Nutting EB, Poe GS. A new combination for bleaching teeth. J S Calif Dent Assoc 1963;31:289-91.

16. Pécora JD, Souza Neto MD, Costa WF. Apresentação de um método químico que revela in vitro a passagem do peróxido de hidrogênio a $30 \%$ através da dentina radicular. Rev Paul Odontol 1991;13(2):34-6.

17. Rotstein I, Friedman S. pH variation among materials used for intracoronal bleaching. J Endod 1991;17(8):376-9.

18. Rotstein I, Lehr Z, Gedalia I. Effect of bleaching agents on inorganic components of human dentin and cementum. J Endod 1992;18(6):290-3.

19. Rotstein I, Toreck Y, Misgav R. Effect of cementum defects on radicular penetration of $30 \% \mathrm{H}_{2} \mathrm{O}_{2}$ during intracoronal bleaching. J Endod 1991;17(5):230-3.

20. Rotstein I, Friendman S, Mor C, Katznelson J, Sommer $\mathrm{M}$, Bab I. Histological characterization of bleaching-induced external resorption in dogs. J Endod 1991;17(9):436-41.

21. Weiger R, Kuhn A, Löst C. Effect of various types of sodium perborate on the $\mathrm{pH}$ of bleaching agents. J Endod $1993 ; 19(5): 239-41$.

Recebido para publicação em 28/01/02 Enviado para reformulação em 14/06/02 Aceito para publicação em 19/06/02 